\title{
Propiedades psicométricas del Cuestionario de Violencia entre Novios en universitarios peruanos
}

\section{Psychometrics properties of the Dating Violence Questionnaire in Peruvian undergraduate students}

\section{Propriedades psicométricas do Questionário de Violência no Noviazgo em estudantes universitários peruanos}

\author{
Romina Raiser Velarde ${ }^{1}$, ORCID 0000-0003-3349-9568 \\ Eduardo Manzanares-Medina ${ }^{2}$, ORCID 0000-0002-3377-311X \\ ${ }^{12}$ Universidad Peruana de Ciencias Aplicadas, Perú
}

\begin{abstract}
Resumen: El objetivo del presente estudio fue determinar las propiedades psicométricas del Cuestionario de Violencia entre Novios (CUVINO) en universitarios de la provincia de Tacna, Perú. La muestra estuvo compuesta por 500 estudiantes de una universidad privada, con edades entre 18 a 35 años. Se aplicó el CUVINO y la Escala de Violencia Contra las Mujeres en relaciones de pareja (VcM). Para evaluar la evidencia de validez basada en la estructura interna del instrumento, se realizó un análisis factorial confirmatorio, del cual se obtuvo como resultado adecuados índices de ajuste para el modelo de ocho factores relacionados $\left(\chi^{2} / \mathrm{gl}=4.574, \mathrm{CFI}=.961, \mathrm{TLI}=.956 \mathrm{SRMR}=.054, \mathrm{RMSEA}=.085\right) . \mathrm{Se}$ calculó la consistencia interna y se obtuvieron valores alfa y omega que oscilan entre .84 y .91. Se utilizó la prueba no paramétrica de Spearman para el análisis de validez convergente. El resultado del análisis de correlaciones entre factores del modelo planteado y la Escala de $\mathrm{VcM}$ fueron que todas son positivas y estadísticamente significativas $(\mathrm{p}<.05)$, con magnitud alta $(\mathrm{rs}>.50)$ y moderada (rs $>.30)$. Se concluye que el CUVINO cuenta con adecuadas propiedades psicométricas para su uso en la muestra de estudio.
\end{abstract}

Palabras clave: violencia; CUVINO; validez; confiabilidad; universitarios. 
Abstract: The objective of the present study was to determine the psychometric properties of the Dating Violence Questionnaire (CUVINO) in peruvian university students, in the province of Tacna, Peru. The sample consisted of 500 students from a private university, aged between 18 and 35 years old. The CUVINO and the Scale of Violence Against Women in relationships were applied $(\mathrm{VcM})$. To evaluate the validity evidence based on the internal structure of the instrument, a confirmatory factor analysis was performed, from which, as a result adequate fit indices were obtained for the model of eight related factors $\left(\chi^{2} / \mathrm{gl}=4.574\right.$, $\mathrm{CFI}=.961$, TLI $=.956, \mathrm{SRMR}=.054$, RMSEA $=.085)$. The internal consistency was calculated and Alpha and Omega were obtained that oscillate between .84 and .91. The Spearman's nonparametric test was used for the convergent validity analysis. The result of the analysis of correlations between the factors of the proposed model and the VcM Scale was that all were positive and statistically significant $(\mathrm{p}<.05)$ with a high $(\mathrm{rs}>.50)$ and moderate ( $\mathrm{rs}>.30$ ) magnitude. It is concluded that CUVINO possess adequate psychometric properties for its use in the study sample.

Keywords: violence; CUVINO; validity; reliability; undergraduate students.

Resumo: O objetivo deste estudo foi determinar as propriedades psicométricas do Questionário de Violência entre Namorados (CUVINO) em estudantes universitários da província de Tacna, Peru. A amostra foi composta por 500 alunos de uma universidade privada, com idades entre 18 e 35 anos. Foram aplicados o CUVINO e a Escala de Violência Contra as Mulheres nos Relacionamentos ( $\mathrm{VcM}$ ). Para avaliar as evidências de validade com base na estrutura interna do instrumento, foi realizada uma análise fatorial confirmatória, que resultou em índices de ajuste adequados para o modelo de oito fatores relacionados $\left(\chi^{2} / \mathrm{gl}=\right.$ $4.574, \mathrm{CFI}=.961, \mathrm{TLI}=.956 \mathrm{SRMR}=.054, \mathrm{RMSEA}=.085)$. Foi calculada a consistência interna e obtiveram-se valores de alfa e ômega variando entre .84 e .91 . O teste não paramétrico de Spearman foi usado para a análise da validade convergente. Os resultados das análises de correlações entre os fatores do modelo proposto e a Escala $\mathrm{VcM}$ foram todos positivos e estatisticamente significativos $(\mathrm{p}<.05)$, com magnitude elevada ( $\mathrm{rs}>.50)$ e moderada ( $\mathrm{rs}>.30$ ). Conclui-se que o CUVINO possui propriedades psicométricas adequadas para utilização na amostra estudada.

Palavras-chave: violência; CUVINO; validade; confiabilidade; universitários.

Recibido: 18/04/2021

Aceptado: 12/10/2021

Cómo citar:

Raiser, R. \& Manzanares-Medina, E. (2021). Propiedades psicométricas del Cuestionario de Violencia entre Novios en universitarios peruanos. Ciencias Psicológicas, 15(2), e-2548. doi: https://doi.org/10.22235/cp.v15i2.2548

Correspondencia: Eduardo Manzanares-Medina, Universidad Peruana de Ciencias Aplicadas, Perú. E-mail: pcpsemam@upc.edu.pe 
En el noviazgo, la violencia es un fenómeno casi imperceptible que puede confundirse con muestras de cariño y de protección (Yanes, Hernández de la Cruz \& Bautista 2019). El maltrato en las parejas es un fenómeno de carácter universal, multidimensional y multicausal, que sigue incrementando a pesar de los esfuerzos que las entidades públicas y privadas han realizado para desarrollar estrategias de prevención y control (Ocampo, 2015). En la actualidad, la violencia en parejas jóvenes supone una importante problemática debido a sus graves consecuencias y al aumento de su prevalencia (Carrascosa, Cava \& Buelga, 2018).

En Latinoamérica durante las últimas décadas esta situación ha generado también un incremento en la conciencia pública sobre el riesgo de experimentar violencia como parte de los vínculos interpersonales más cercanos. Si bien las relaciones interpersonales íntimas son un espacio para vivir amor y aceptación, también puede ser un espacio para propiciar poder y violencia (Rodríguez, Riosvelasco \& Castillo, 2018). La Organización Mundial de la Salud (OMS) considera que la violencia es un problema de salud pública a partir de inicios del presente siglo. A nivel mundial se estima que entre el $10 \%$ y el $69 \%$ de las mujeres han sufrido alguna forma de maltrato (psicológico, físico o sexual) por parte de su compañero (OMS, 2002). La violencia de pareja es el tipo más común de violencia contra la mujer, ya que afecta al $30 \%$ de las mujeres en todo el mundo (OMS, 2013).

En Perú, según las cifras reportadas por el Instituto Nacional de Estadística e Informática (INEI, 2019), en el año 2018, el 63.2 \% de las mujeres entre 15 a 49 años sufrieron algún tipo de violencia ejercida por su pareja. Dentro de este grupo, el $58.9 \%$ fueron víctimas de violencia psicológica, el $30.7 \%$ agredidas físicamente y el $6.8 \%$ fueron violentadas de manera sexual. El Ministerio de la Mujer y Poblaciones Vulnerables (MIMP), atendió 133697 víctimas de violencia familiar y sexual en el año 2018 (INEI, 2019). En el siguiente año, según el MIMP (2019), se atendieron 181885 casos de violencia contra la mujer, violencia familiar y sexual de enero a diciembre en los Centros de Emergencia Mujer (CEM) a nivel nacional, dentro de los cuales fueron considerados como víctimas a un $85 \%$ de mujeres y $15 \%$ de hombres.

Diversas investigaciones muestran cifras elevadas de violencia en departamentos del Perú, siendo Tacna uno de los más afectados. El MIMP reportó 1485 casos registrados de violencia, siendo $86 \%$ víctimas mujeres y $14 \%$ varones agredidos en los CEM (La República, 2019). Asimismo, según la Encuesta Demográfica y de Salud Familiar (ENDES), Tacna evidencia ser uno de los departamentos del Perú con un alto índice de violencia y cuenta con escasas investigaciones. Un estudio acerca de la violencia contra las mujeres y los integrantes del grupo familiar en Tacna dio como resultado que ocupa el octavo lugar en cuanto a índices de violencia física, y décimo segundo en violencia sexual en el país. La prevalencia de violencia en el año 2017 en mujeres por parte de su pareja fue de $51.4 \%$. El tipo de violencia más frecuente fue el psicológico (45.5\%), seguido del físico $(26.9 \%)$ y sexual $(4.9 \%)$. Las razones por las que las víctimas no buscan ayuda son porque no lo consideran necesario (47.7\%), no saben a dónde ir (16.7\%), tienen miedo a que les golpeen de nuevo $(9.2 \%)$, miedo de causarle problemas a su agresor $(8.5 \%)$, de nada sirve $(5.7 \%)$ y otras razones $(12.2 \%$ ) (ENDES, 2018, citado en Observatorio Nacional de la Violencia contra las Mujeres y los Integrantes del Grupo Familiar, 2018).

Todos estos datos ponen a la luz la problemática vinculada a la violencia de género, la cual se define como un conjunto de actos que provocan daño corporal y abuso psicológico, que puede darse en privado o situaciones sociales (Rodríguez et al., 2010). Se considera 
violencia en las relaciones de noviazgo a cualquier tipo de agresión intencionada de un miembro de la pareja contra el otro durante la relación (Rubio, López, Carrasco \& Amor, 2017). Es decir, actos que lastiman a la otra persona (Rodríguez et al., 2018).

Como parte de dicho contexto general, cabe recalcar la dificultad que tienen los adolescentes y jóvenes para reconocer que son víctimas del maltrato (Díaz et al., 2013). Uno de los aspectos que aumenta la incapacidad de reconocer la violencia durante el noviazgo en esta etapa es la idealización que adolescentes y jóvenes realizan de las conductas violentas con base en el amor romántico, así como restarle importancia a comportamientos violentos como son los celos y el control obsesivo. Ambas razones serían una justificación para continuar con la pareja, lo cual impide que reconozcan un ambiente hostil dentro de una relación (Gómez, Delgado \& Gómez, 2014).

La violencia en el noviazgo es un fenómeno que puede seguir un patrón bidireccional, sin embargo, por lo general se considera al hombre como victimario y a la mujer como víctima, sin considerar que ambos pueden desempeñar ambos roles (Del Ángel \& Barraza, 2017). Bajo esta perspectiva, tanto hombres y mujeres que forman parte de una relación bajo un contexto de violencia son un par de seres humanos sujetos de derechos, que deben enfrentar las consecuencias de sus actos y recibir ayuda profesional (Beltrán, Albán, Zumba, Vera \& Figueroa, 2018).

Como parte de las relaciones de parejas que tienen una dinámica donde está presente la violencia, se han identificado ocho tipos de abuso: (a) desapego, considerado una actitud de indiferencia hacia la pareja y sus sentimientos; (b) humillación, definido como críticas personales contra la autoestima y orgullo personal; (c) sexual, incluye conductas sexuales no deseados por la pareja; (d) coerción, es la presión ejercida sobre alguien para forzar su voluntad o conducta a través de amenazas o manipulaciones; (e) castigo físico, en el cual la persona ejecuta golpes o daña objetos con significado emocional para la víctima; (f) violencia basada en género, sería la desestimación de la condición de mujer/hombre; (g) castigo emocional, que son demostraciones de enfado con el objetivo de manipular a la pareja; (h) instrumental, en el cual la persona usa medios indirectos para infligir daños o sufrimiento en la víctima (Cortés-Ayala et al., 2015).

Se destaca la necesidad de seguir estudiando el fenómeno de la violencia en la pareja y sus problemáticas asociadas, para lo cual se requiere de instrumentos que permitan su apropiada medición. Dentro de la revisión de la literatura se han encontrado algunos instrumentos enfocados en medir el maltrato en pareja, pero que no evalúan de manera específica la experiencia ocurrida durante el noviazgo. Por ejemplo, en el 2005, Langhinrichsen-Rohling señaló la aparición de la Conflict Tactics Scale (CTS; Straus, 1979), como uno de los primeros instrumentos para evaluar de manera cuantitativa la agresión ocurrida entre las relaciones interpersonales de convivencia. Este instrumento ofrece información solo acerca de dos formas de agresión: física y verbal. La CTS-2 (Straus, 2008) incluyó dos escalas nuevas: coerción sexual y gravedad de las lesiones. El Index of Supose Abuse (ISA; Hudson \& Mcintosh, 1981) contiene dos subescalas: física y no física, de un modo similar a la CTS original y no evalúa de manera específica las relaciones de pareja. Adicionalmente, otro instrumento considerado útil fue el Conflict in Adolescent Dating Relationships Inventory (CADRI; Fernández, Fuertes \& Pulido, 2006) que ofrece seis subescalas que cubren y mejoran los criterios físicos, psicológicos y sexuales, y está diseñado para población adolescente y joven. Sin embargo, la validación llevada a cabo en España 
aporta datos acerca de las agresiones realizadas y no de la experiencia en sí (Rodríguez et al., 2010).

Cabe resaltar que ninguno de los instrumentos descritos fue diseñado específicamente para parejas jóvenes y las validaciones con este rango de edad parecen obedecer más a una cuestión de accesibilidad a los estudiantes universitarios que al interés por obtener información acerca de cómo es que se llevan a cabo sus relaciones de pareja. Por ende, el Cuestionario de Violencia Entre Novios (CUVINO) es un instrumento que cubre los vacíos señalados en cuanto a la medición de la violencia en el noviazgo de jóvenes, el cual fue validado en España, México y Argentina en el 2010 y en español en una muestra de 5170 participantes. Es específico para la evaluación de la violencia sufrida dentro de las relaciones de pareja, diseñado partiendo de información ofrecida por adolescentes y jóvenes de ambos sexos, y ha sido revisado a través de experiencias piloto llevadas a cabo en población juvenil. Cuenta con 42 ítems conductuales que deben ser contestados en formato Likert (entre 0 y 4 ) y ocho dimensiones: desapego, humillación, sexual, coerción, físico, género, castigo emocional e instrumental, las cuales han sido descritas previamente.

En la validación transcultural del CUVINO en España, México y Argentina, la estructura factorial mostró un total de 8 tipos de abuso con una varianza explicada del $51.3 \%$. La estructura encontrada resultó similar a la propuesta inicialmente por Rodríguez, Antuña, Rodríguez-Díaz y Herrero (2007); sin embargo, el ítem 20 ("Ha lanzado objetos contundentes contra ti") que en el primer estudio se encontraba en el factor violencia instrumental fue asignado a violencia física. La fiabilidad total de la escala (alfa de Cronbach de .93) y de las subescalas alfas comprendidos entre .58 y .81 indican datos psicométricos para considerar el CUVINO como herramienta válida y confiable.

A partir de lo mencionado previamente, se considera que el presente estudio tiene un aporte instrumental pues permitirá revisar la calidad psicométrica de un instrumento para medir la violencia en relaciones de pareja en muestra de jóvenes peruanos. En cuanto al valor práctico, será una herramienta útil para medir los efectos de programas de prevención que podrían aplicarse con respecto a violencia entre parejas. Además, es socialmente relevante ya que esta herramienta brinda una alternativa de evaluación de violencia entre parejas, la cual sería útil para prevenir y hacer frente a las relaciones interpersonales afectivas que padecen de abusos y maltratos.

De esta manera, el objetivo general de la investigación es determinar las propiedades psicométricas de la escala CUVINO. Con respecto a los objetivos específicos, se busca determinar la evidencia de validez basada en la estructura interna, establecer evidencias de validez convergente mediante la relación de los puntajes obtenidos del CUVINO y la Escala de Violencia Contra las Mujeres en relaciones de pareja (VcM; Vara-Horna \& López-Odar, 2016) y, por último, estimar la confiabilidad por consistencia interna.

\section{Materiales y método}

\section{Participantes}

Participaron de este estudio 500 estudiantes universitarios de los cuales $46.8 \%$ son mujeres y $53.2 \%$ hombres, con un rango de edad entre 18 y 35 años $(\mathrm{M}=24.10, \mathrm{DE}=2.70)$, de diversas carreras $(13.6 \%$ de psicología, $7.2 \%$ de derecho, $13.8 \%$ de ingeniería, $11.8 \%$ de educación, $20.6 \%$ de negocios, $7.6 \%$ de hotelería y turismo, $5.2 \%$ de comunicación, 
$10.8 \%$ de contabilidad, $4.6 \%$ de ingeniería y $4.8 \%$ de medicina). El $80.8 \%$ mantienen una relación de pareja actual, mientras que el $19.2 \%$ han mantenido una relación de pareja en el pasado. Esta muestra ha sido elegida sobre la base de algunos criterios acerca del tamaño de la muestra en estudios instrumentales, considerando 50 muy deficiente; 100 deficiente; 200 aceptable; 300 bueno; 500 muy bueno, 1000 o más excelente (Comrey \& Lee, 1992, citado en Lloret-Segura, Ferreres-Traver, Hernández-Baeza \& Tomás-Marco, 2014).

Los participantes fueron seleccionados a través de un muestreo no probabilístico, (Hernández, Fernández \& Baptista, 2014). La técnica de muestreo fue por conveniencia, dada la accesibilidad y disponibilidad de los participantes. Como criterios de inclusión se consideraron los siguientes: personas nacidas en el Perú que estén residiendo en Tacna, que tengan o hayan tenido pareja y acepten participar voluntariamente en este estudio.

\section{Instrumentos}

Cuestionario de Violencia Entre Novios (CUVINO). Para la presente investigación, el CUVINO fue revisado por tres expertos y se determinó que no habría que realizar ninguna adaptación en el lenguaje. Ha sido validado en España, México y Argentina. Su objetivo es evaluar las relaciones interpersonales en las parejas e identificar la existencia o no de violencia dentro de estas. El CUVINO es específico para la evaluación de violencia recibida dentro de las relaciones de pareja y puede ser aplicado en jóvenes y adultos. Está compuesto por 42 ítems los cuales están divididos en 8 dimensiones: desapego (ítems 6, 14, 22, 30, 32, 33 y 37), humillación $(7,15,23,31,36,40$ y 41), sexual $(2,10,18,26,34$ y 39), coerción (1, $9,17,25,38$ y 42), físico $(5,13,20,21$ y 29), género $(3,11,19,27$ y 35), castigo emocional $(8,16$ y 24$)$ e instrumental $(4,12$ y 28$)$. Los ítems deben ser respondidos en un formato Likert de frecuencia de cinco opciones $(0=$ nunca y $4=$ muy frecuentemente $)$. Las cargas factoriales de los ítems oscilan entre .37 y .77 . En cuanto a la confiabilidad por consistencia interna los valores alfa oscilan entre .68 y .82 (Rodríguez et al., 2010).

Escala de Violencia Contra las Mujeres. Se utilizó la Escala de Violencia Contra las Mujeres en relaciones de pareja (VcM, Vara-Horna \& López-Odar, 2016) con el propósito de determinar evidencias de validez convergente mediante la relación de los puntajes obtenidos del CUVINO y la VcM. Esta escala posee 14 ítems que miden indicadores de violencia psicológica, económica, física leve, física grave, sexual y daños ejercidos por la pareja actual o expareja. La violencia psicológica hace referencia a las acciones u omisiones ejercidas por el agresor con el fin de controlar la conducta o reducir la autonomía de la pareja, como ataques verbales, insultos, humillaciones, abandono y amenazas. La violencia económica implica el control de los recursos financieros o bienes materiales utilizando la fuerza física para lograrlo. La violencia física leve incluye golpes, empujones, cachetadas y ataques contra algunas partes del cuerpo. Mientras que la violencia física grave implica el uso de objetos punzocortantes, contundentes o armas de fuego. La violencia sexual son actos realizados contra la libertad sexual, como la violación y tocamientos indebidos. En cuanto a los daños, es en caso el agresor haya generado moretones graves, esguinces, fracturas o lesiones. Cada ítem evalúa el nivel de intensidad del ataque y tiene alternativas de respuestas con valores de interpretación: nunca, pasó antes, ahora no, una o dos veces, entre 3 a 5 veces, entre 6 y 10 veces, entre 11 a 20 veces y más de 20 veces. Está escala ha sido diseñada y utilizada para estimar el nivel de violencia en mujeres que trabajan en grandes y medianas 
empresas y en microempresas. Con la escala se puede determinar la violencia ocurrida en el último año y anterior al último año (Vara-Horna \& López-Odar, 2016). Los valores de fiabilidad mediante el alfa de Cronbach oscilan entre .65 y .83 ; rho entre .65 y .83 y la fiabilidad compuesta entre .85 y .90 . En cuanto a la validez basada en la estructura interna, el porcentaje de varianza explicada de los factores es superior al $50 \%$ y los pesos factoriales superiores a .71. Para el presente estudio los valores de confiabilidad por consistencia interna, mediante el coeficiente omega, oscilaron entre .77 y .92, indicando una adecuada confiabilidad.

\section{Procedimiento}

Se solicitó y obtuvo el permiso de los autores de las pruebas que se aplicaron y se tramitó el permiso necesario para acceder a una institución universitaria privada de Tacna para realizar la recolección de la información. Obtenidos los permisos correspondientes, se aplicaron los cuestionarios a los estudiantes dentro de las aulas, acordando previamente con los profesores y preguntando a los estudiantes si estarían dispuestos a llenar los cuestionarios de manera voluntaria. Se entregó a los participantes el consentimiento informado y una ficha sociodemográfica.

Los cuestionarios fueron aplicados por la investigadora en un tiempo aproximado de 30 a 45 minutos. Se realizó una pequeña descripción del cuestionario en el comentario adjunto para que pudieran realizarlo de manera adecuada y se comunicó que solo será utilizado para temas relacionados a la investigación. Se aclaró la participación voluntaria y el carácter confidencial del estudio, así como el anonimato en las respuestas.

\section{Diseño y análisis de datos}

El presente estudio es de tipo instrumental (Ato, López \& Benavente, 2013).

Se hizo una revisión de los protocolos y se desecharon aquellos en los que hubiese alguna omisión de datos en la ficha sociodemográfica o cuestionarios. Se realizó el análisis de datos utilizando los programas estadísticos Jamovi y RStudio, a los cuales se exportó la base de datos en una hoja de Excel. Se realizó el análisis de ítems, calculando la media, desviación estándar, el valor de la asimetría y la curtosis de todos los ítems ( $\mathrm{g}_{1}$ y g2 $>|1|$ ); así como el valor de la correlación ítem-total corregida para cada una de las dimensiones ( $r_{i t c}<$ .20). Para el análisis factorial confirmatorio se utilizó el software RStudio y el paquete Lavaan (Rosseel, 2012). Se empleó el método de estimación Mínimos Cuadrados Ponderados Robustos (WLSMV), recomendado cuando se trabaja con datos categóricos y no se cumple la normalidad en la distribución de los ítems (Li, 2016). Por las razones mencionadas anteriormente, las estimaciones del AFC se trabajaron sobre la base de la matriz de correlaciones policóricas.

Para evaluar el ajuste se tomaron en cuenta los siguientes índices. En primer lugar, los índices analizados para comparar los modelos fueron el chi-cuadrado entre los grados de libertad $\left(\chi^{2} / g l,\right)$, el índice de ajuste comparativo (CFI), la raíz del residuo cuadrático promedio estandarizado (SRMR), el índice de aproximación de la raíz de cuadrados medios del error (RMSEA) y el criterio de índice de ajuste no normalizado o índice de Tucker- Lewis (TLI).

En cuanto a la utilidad específica, el chi-cuadrado entre los grados de libertad $\left(\chi^{2} / \mathrm{gl}\right.$ <3) comprueba la significancia del modelo, contrastando la hipótesis nula de que todos los errores del modelo son nulos; sin embargo, al ser sensible al tamaño muestral, se compara 
con los grados de libertad (Ruiz, Pardo \& San Martín, 2010). El índice de ajuste comparativo (CFI) indica un buen ajuste del modelo para valores próximos a 1. Se considera que para ser aceptable debe oscilar en valores mayores o iguales a .90 y a partir de .95 , se consideran ajustes excelentes (Wu, Li \& Zumbo, 2007). En la raíz del residuo cuadrático promedio estandarizado (SRMR) se aconsejan valores menores o cercanos a .05 para indicar un buen ajuste del modelo (Byrne, 2006). Para la raíz cuadrada del error medio cuadrático (RMSEA) valores inferiores a .08 son indicativos de un buen ajuste (Medrano \& Muñoz, 2017). Por último, el índice de ajuste no normalizado (TLI) compara el ajuste por grados de libertad del modelo propuesto y nulo. Este índice tiende a 1 para modelos con muy buen ajuste, considerándose aceptables valores superiores a .90 (Medrano \& Muñoz, 2017).

Se determinó la evidencia de la validez basada en la relación con otra variable mediante el programa Jamovi. Para ello se relacionó el puntaje obtenido en el CUVINO con el puntaje obtenido en la VcM. Para la interpretación de la magnitud de las correlaciones se tomó en cuenta el criterio propuesto por Cohen (1988): .10 a .29 (baja), .30 a .49 (moderada) y .50 a más (alta). Finalmente, se estimó la confiabilidad basada en la consistencia interna del instrumento con el coeficiente omega $(\omega>.70)$.

\section{Resultados}

\section{Análisis descriptivo y de homogeneidad de los ítems}

Se calcularon los estadísticos de tendencia central y de dispersión de los ítems (tabla 1), en donde los índices de asimetría fueron inferiores a |1|, pero se observaron valores mayores en la curtosis (Lloret-Segura et al., 2014). Posteriormente, se analizó la homogeneidad de los ítems del CUVINO mediante las correlaciones ítem-test corregidas $\left(r_{i t c}\right)$, donde se obtuvieron valores superiores a .20 (Kline, 1986). El rango de las $r_{i t c}$ de los ítems estuvo entre .62 y .81, razón por la cual se mantuvieron todos los ítems para el análisis factorial. 
Tabla 1.

Estadísticos descriptivos y correlaciones ítem-tes c corregidas del CUVINO

\begin{tabular}{|c|c|c|c|c|c|c|c|}
\hline Ítem & Min. & Máx. & $M$ & $D E$ & $g_{I}$ & $g_{2}$ & $r_{i t c}$ \\
\hline 1 & 0 & 4 & 1.56 & 1.26 & .35 & -1.06 & .69 \\
\hline 2 & 0 & 4 & 1.62 & 1.25 & .21 & -1.08 & .74 \\
\hline 3 & 0 & 4 & 1.75 & 1.26 & .17 & -1.08 & .74 \\
\hline 4 & 0 & 4 & 1.30 & 1.34 & .55 & -1.09 & .71 \\
\hline 5 & 0 & 4 & 1.66 & 1.40 & .20 & -1.35 & .81 \\
\hline 6 & 0 & 4 & 1.89 & 1.24 & -.01 & -1.06 & .73 \\
\hline 7 & 0 & 4 & 1.45 & 1.32 & .37 & -1.19 & .74 \\
\hline 8 & 0 & 4 & 1.85 & 1.38 & .05 & -1.28 & .69 \\
\hline 9 & 0 & 4 & 1.80 & 1.35 & .13 & -1.26 & .75 \\
\hline 10 & 0 & 4 & 1.60 & 1.31 & .28 & -1.12 & .72 \\
\hline 11 & 0 & 4 & 1.60 & 1.31 & .25 & -1.16 & .69 \\
\hline 12 & 0 & 4 & 1.34 & 1.40 & .59 & -1.06 & .71 \\
\hline 13 & 0 & 4 & 1.66 & 1.42 & .25 & -1.34 & .80 \\
\hline 14 & 0 & 4 & 1.67 & 1.30 & .28 & -1.05 & .77 \\
\hline 15 & 0 & 4 & 1.65 & 1.35 & .23 & -1.20 & .81 \\
\hline 16 & 0 & 4 & 1.76 & 1.44 & .19 & -1.38 & .80 \\
\hline 17 & 0 & 4 & 1.58 & 1.48 & .35 & -1.36 & .78 \\
\hline 18 & 0 & 4 & 1.77 & 1.40 & .12 & -1.32 & .80 \\
\hline 19 & 0 & 4 & 1.62 & 1.42 & .29 & -1.31 & .75 \\
\hline 20 & 0 & 4 & 1.38 & 1.39 & .55 & -1.09 & .74 \\
\hline 21 & 0 & 4 & 1.45 & 1.36 & .43 & -1.20 & .77 \\
\hline 22 & 0 & 4 & 1.69 & 1.21 & .07 & -1.15 & .77 \\
\hline 23 & 0 & 4 & 1.70 & 1.35 & .12 & -1.30 & .83 \\
\hline 24 & 0 & 4 & 1.75 & 1.36 & .12 & -1.29 & .78 \\
\hline 25 & 0 & 4 & 1.99 & 1.34 & -.10 & -1.26 & .64 \\
\hline 26 & 0 & 4 & 1.66 & 1.31 & .21 & -1.12 & .74 \\
\hline 27 & 0 & 4 & 1.65 & 1.30 & .20 & -1.17 & .77 \\
\hline 28 & 0 & 4 & 1.31 & 1.32 & .60 & -.97 & .67 \\
\hline 29 & 0 & 4 & 1.43 & 1.36 & .34 & -1.34 & .76 \\
\hline 30 & 0 & 4 & 1.84 & 1.37 & .03 & -1.28 & .70 \\
\hline 31 & 0 & 4 & 1.67 & 1.27 & .06 & -1.26 & .75 \\
\hline 32 & 0 & 4 & 1.70 & 1.31 & .06 & -1.26 & .71 \\
\hline 33 & 0 & 4 & 1.80 & 1.25 & .20 & -1.06 & .77 \\
\hline 34 & 0 & 4 & 1.58 & 1.24 & .16 & -1.11 & .75 \\
\hline 35 & 0 & 4 & 1.37 & 1.26 & .35 & -1.22 & .70 \\
\hline 36 & 0 & 4 & 1.48 & 1.31 & .35 & -1.12 & .62 \\
\hline 37 & 0 & 4 & 1.69 & 1.36 & .07 & -1.35 & .76 \\
\hline 38 & 0 & 4 & 1.76 & 1.32 & .07 & -1.25 & .68 \\
\hline 39 & 0 & 4 & 1.47 & 1.21 & .30 & -.97 & .69 \\
\hline 40 & 0 & 4 & 1.39 & 1.22 & .38 & -1.11 & .72 \\
\hline 41 & 0 & 4 & 1.53 & 1.22 & .14 & -1.17 & .74 \\
\hline 42 & 0 & 4 & 1.70 & 1.21 & .04 & -1.04 & .72 \\
\hline
\end{tabular}

Nota $: \mathrm{g}_{1}=$ asimetría; $\mathrm{g}_{2}=$ curtosis $; \mathrm{r}_{\mathrm{itc}}=$ correlación ítem-test corregida. 


\section{Evidencia de validez basada en la estructura interna}

Para investigar la evidencia de validez relacionada a la estructura interna, se realizó un análisis factorial confirmatorio (tabla 2) con el fin de someter los hallazgos previos a un método más robusto. Se probó el modelo original de la prueba para evaluar si se ajustaba de manera adecuada a los datos.

Como parte del primer análisis se obtuvieron los siguientes resultados: el $\chi^{2} / g l=4.938$ $(p<.001)$, el CFI $=.952, \mathrm{TLI}=.948, \mathrm{SRMR}=.059, \mathrm{RMSEA}=.089$. Debido a que los índices no alcanzaron los valores esperados en algunos índices, se analizaron índices de modificación para obtener una mejora del ajuste del modelo.

Con respecto a dichas modificaciones, se especificaron los errores correlacionados entre los ítems 40-41 (humillación = .66) y 20-21 (físico = .54). En segundo lugar, se analizaron los índices referidos a la ubicación de los ítems en las dimensiones, de los cuales se eliminaron el 17, 29, 30 y 14. Con respecto a los tres primeros, se pudo observar que su carga se mostraba relacionada en más de un factor. Con respecto al último ítem, se observó una redacción confusa. Con estas nuevas especificaciones, los nuevos índices obtenidos fueron: $\chi^{2} / g l=4.574(p<.001), \mathrm{CFI}=.961, \mathrm{TLI}=.956 \mathrm{SRMR}=.054, \mathrm{RMSEA}=.085$ $(\mathrm{IC} 90 \%=.82, .88)$.

Las cargas factoriales que presentaron los ítems oscilaron entre .75 y .83 (desapego), .67 y .88 (humillación), .74 y .84. (sexual), .74 y .86. (coerción), .81 y .87 (físico), .74 y .82. (género), .77 y .88 (castigo emocional) y .82 y .87 (instrumental). En todos los casos las cargas factoriales obtenidas son consideradas aceptables, pues superan el valor mínimo recomendable por Tabachnick y Fidell (2013) de .30. 
Tabla 2.

Matriz factorial con cargas estandarizadas del CUVINO

\begin{tabular}{|c|c|c|c|c|c|c|c|c|}
\hline \multirow{2}{*}{ Ítem } & \multicolumn{8}{|c|}{ Factor } \\
\hline & DES & HUM & SEX & $\mathrm{COE}$ & FIS & GEN & CAE & INS \\
\hline 22 & .83 & & & & & & & \\
\hline 33 & .83 & & & & & & & \\
\hline 37 & .80 & & & & & & & \\
\hline 06 & .78 & & & & & & & \\
\hline 32 & .75 & & & & & & & \\
\hline 23 & & .88 & & & & & & \\
\hline 15 & & .86 & & & & & & \\
\hline 07 & & .79 & & & & & & \\
\hline 31 & & .79 & & & & & & \\
\hline 40 & & .77 & & & & & & \\
\hline 41 & & .77 & & & & & & \\
\hline 36 & & .67 & & & & & & \\
\hline 18 & & & .84 & & & & & \\
\hline 26 & & & .80 & & & & & \\
\hline 34 & & & .79 & & & & & \\
\hline 02 & & & .77 & & & & & \\
\hline 10 & & & .76 & & & & & \\
\hline 39 & & & .74 & & & & & \\
\hline 09 & & & & .86 & & & & \\
\hline 42 & & & & .81 & & & & \\
\hline 01 & & & & .79 & & & & \\
\hline 38 & & & & .77 & & & & \\
\hline 25 & & & & .74 & & & & \\
\hline 05 & & & & & .87 & & & \\
\hline 13 & & & & & .87 & & & \\
\hline 21 & & & & & .82 & & & \\
\hline 20 & & & & & .81 & & & \\
\hline 27 & & & & & & .82 & & \\
\hline 19 & & & & & & .82 & & \\
\hline 03 & & & & & & .80 & & \\
\hline 35 & & & & & & .78 & & \\
\hline 11 & & & & & & .74 & & \\
\hline 16 & & & & & & & .88 & \\
\hline 24 & & & & & & & .84 & \\
\hline 08 & & & & & & & .77 & \\
\hline 12 & & & & & & & & .87 \\
\hline 04 & & & & & & & & .86 \\
\hline 28 & & & & & & & & .82 \\
\hline
\end{tabular}

Nota : DES = desapego; HUM = humillación; $\mathrm{SEX}=$ sexual; $\mathrm{COE}=$ coerción; FIS = físico; $\mathrm{GEN}=$ género; $\mathrm{CAE}=$ castigo emocional; $\mathrm{INS}=$ Instrumental. 


\section{Evidencias de validez basada en relaciones con otras variables}

Se realizó un análisis de normalidad por medio de la prueba de Kolmogorov-Smirnov (K-S), tomando como referencia el tamaño de la muestra $(N>50)$, con lo que se encontró que ninguno de los factores presenta una distribución normal $(p<.05)$, razón por la cual se determinó usar la prueba no paramétrica de Spearman para el análisis de validez convergente.

Se realizó el análisis de correlaciones entre factores con la finalidad de establecer el grado de intensidad entre las relaciones existentes entre los factores del modelo planteado y la escala de la VcM. Se observa que la dimensión instrumental del CUVINO presentó correlaciones significativas y moderadas con las dimensiones de VcM. Por otro lado, se aprecia que las dimensiones de violencia económica y física grave presentaron correlaciones significativas y moderadas con las dimensiones del CUVINO. En todos los demás casos las correlaciones obtenidas fueron significativas $(p<.05)$ y de magnitud alta $\left(r_{s}>.50\right.$; tabla 3$)$.

Tabla 3.

Estadísticos descriptivos y matriz de correlaciones entre las dimensiones del CUVINO y VcM

\begin{tabular}{|c|c|c|c|c|c|c|c|c|}
\hline \multirow{2}{*}{ Dimensión } & \multirow{2}{*}{$M$} & \multirow{2}{*}{$D E$} & \multicolumn{6}{|c|}{ VcM } \\
\hline & & & PSI & ECO & VFL & VFG & SEX & DAÑ \\
\hline \multicolumn{9}{|l|}{ CUVINO } \\
\hline DES & 8.92 & 5.03 & $.55 * *$ & $.36 * *$ & $.54 * *$ & $.39 * *$ & $.53 * *$ & $.54 * *$ \\
\hline HUM & 11.33 & 7.08 & $.53 * *$ & $.40 * *$ & $.59 * *$ & $.46^{* *}$ & $.50 * *$ & $.54 * *$ \\
\hline SEX & 10.10 & 5.96 & $.53 * *$ & $.42 * *$ & $.56 * *$ & $.48 * *$ & $.59 * *$ & $.54 * *$ \\
\hline $\mathrm{COE}$ & 9.18 & 5.06 & $.61 * *$ & $.34 * *$ & $.59 * *$ & $.39 * *$ & $.50 * *$ & $.50 * *$ \\
\hline FIS & 6.41 & 4.78 & $.55^{* *}$ & $.36 * *$ & $.58 * *$ & $.50 * *$ & $.51 * *$ & $.54 * *$ \\
\hline GEN & 8.32 & 5.15 & $.51 * *$ & $.40 * *$ & $.59 * *$ & $.46^{* *}$ & $.57 * *$ & $.55^{* *}$ \\
\hline CAE & 5.58 & 3.55 & $.61 * *$ & $.35 * *$ & $.56 * *$ & $.43 * *$ & $.51 * *$ & $.50 * *$ \\
\hline INS & 4.10 & 3.51 & $.33 * *$ & $.46^{* *}$ & $.39 * *$ & $.49 * *$ & $.42 * *$ & $.45^{* *}$ \\
\hline$M$ & - & - & 10.97 & 2.79 & 4.01 & 2.98 & 3.17 & 3.21 \\
\hline$D E$ & - & - & 6.02 & 1.55 & 2.53 & 1.79 & 1.99 & 1.94 \\
\hline
\end{tabular}

Nota: DES = desapego; HUM = humillación; $\mathrm{SEX}=$ sexual; $\mathrm{COE}=$ coerción; FIS = físico; $\mathrm{GEN}$ = género; $\mathrm{CAE}=$ castigo emocional; INS = instrumenta; $\mathrm{PSI}=$ psicológico $; \mathrm{ECO}=$ económica; $\mathrm{VFL}=$ física leve; $\mathrm{VFG}=$ física grave; $\mathrm{SEX}=$ sexual; $\mathrm{DAN}=$ daño. $* * p<.01$ 


\section{Evidencia de confiabilidad}

Para la evidencia de confiabilidad se calculó la consistencia interna a partir del coeficiente omega. Se tomó como referencia que una adecuada confiabilidad debe ser mayor a .70 para considerarse aceptable (Viladrich, Angulo-Brunet \& Doval, 2017). Se obtuvieron valores del coeficiente omega que oscilan entre .84 y .91, los cuales son considerados aceptables (tabla 4).

Tabla 4.

Consistencia interna de las puntuaciones del CUVINO

\begin{tabular}{lc}
\hline Dimensión & $\omega$ \\
\hline Desapego & $0.91($ IC 95\%: .89, .92) \\
Humillación & $0.91($ IC 95\%: .89, .92) \\
Sexual & 0.89 (IC 95\%: .87, .91) \\
Coerción & 0.89 (IC 95\%: .87, .91) \\
Físico & $0.90($ IC 95\%: .88,.92) \\
Género & $0.90($ IC 95\%: .88, .92) \\
Castigo emocional & 0.84 (IC 95\%: .81, .86) \\
Instrumental & 0.84 (IC 95\%: .81, .86) \\
\hline
\end{tabular}

\section{Discusión}

Como resultado del análisis factorial realizado y de la consistencia interna de sus dimensiones se confirmó la estructura interna encontrada en el instrumento original (ocho factores). Se obtuvieron valores del coeficiente omega que oscilan entre .84 y .91

Los índices de ajuste del análisis factorial confirmatorio realizado fueron: $\chi^{2} / g l=$ $4.937, p<.001 ; \mathrm{CFI}=.952 ; \mathrm{SRMR}=.059 ; \mathrm{RMSEA}=.089 ; \mathrm{TLI}=.948$. Debido a que los índices no alcanzaron los valores esperados, se analizaron índices de modificación. A diferencia de la propuesta por Rodríguez et al. (2010), en este estudio se analizaron índices de modificación y errores correlacionados entre los ítems 40 y 41 (humillación) y 20 y 21 (físico). Estos ítems mostraban cierta similitud en la redacción. Además, al ser ítems consecutivos, podrían haber generado estos resultados (Domínguez-Lara, 2019). También se analizaron los índices referidos a la ubicación de los ítems en las dimensiones, de los cuales se eliminaron el 17, 29, 30 y 14. Con respecto a los tres primeros, se pudo observar que sus cargas se mostraron en más de un factor (Blum, Auné, Ahumada, Galibert \& Attorresi, 2013). Con respecto al ítem 14, se observó una redacción en donde se utiliza la negación que suele confundir a quienes responden (Muñiz, 2018). 
Con estas nuevas especificaciones, los nuevos índices obtenidos fueron $\chi^{2} / g l=4.574$, $\mathrm{CFI}=.961, \mathrm{TLI}=.956 \mathrm{SRMR}=.054 \mathrm{y}$ RMSEA $=.085(\mathrm{IC} 90 \%=.82, .88)$. Estos resultados indican que en la mayoría de los índices llegaron al valor esperado según la literatura especializada. Si bien es cierto el índice de ajuste absoluto $\left(\chi^{2} / g l\right)$ no alcanzó el valor recomendado, autores como Abad, Olea, Ponsoda y García (2011) desaconsejan su uso y advierten tener cuidado en su interpretación debido a que sigue siendo sensible al tamaño muestral, incluso si la discrepancia entre el chi cuadrado y los grados de libertad es pequeña. Adicionalmente, el RMSEA no alcanzó el criterio esperado según el consenso de la literatura para considerar un ajuste aceptable (<.08). Sin embargo, autores como Keith (2015) señalan que dentro de la evaluación de los modelos lo que indicaría un ajuste inaceptable sería encontrar valores por encima del .10.

Cabe mencionar que los resultados del análisis factorial confirmatorio han sido obtenidos por el uso del método de estimación Mínimos Cuadrados Ponderados Robustos (WLSMV), el cual es recomendado utilizar cuando se trabaja con ítems que no presentan normalidad (Li, 2016). Es decir, el tipo de muestreo no se limitó a una población con características determinadas. Por ende, la mayor cantidad en el patrón de respuestas han sido concentradas en las opciones más bajas del instrumento (asimetría positiva).

Con respecto a la evidencia de confiabilidad, se calculó la consistencia interna y se obtuvieron valores omega que oscilan entre .84 y .91, los cuales son considerados aceptables (Viladrich et al., 2017). Se reportaron valores para cada factor de violencia recibida. Con respecto a los coeficientes omega, para el factor humillación se obtuvo (.91 y .91), sexual (.89 y .89), coerción (.88 y .89), físico (.90 y .90), género (.90 y .90). y para castigo emocional (.84 y .84.) respectivamente. Dichos valores son superiores a la propuesta por Rodríguez et al. (2010), en donde se encuentran valores alfa entre .58 y .81. Probablemente, esto es dado ya que en el estudio de Rodríguez et al. (2010) existieron casos atípicos o de respuesta inconsistente, los cuales no fueron contemplados debido a la amplitud de su muestra. Además, en el estudio original se calcularon coeficientes de confiabilidad alfa, sin haber evaluado los supuestos para su uso (igualdad de cargas factoriales y ausencia de errores correlacionados), motivo por el cual los valores de confiabilidad pudieron haberse infraestimado.

En cuanto a los resultados del análisis de validez convergente entre los factores del modelo planteado y la escala de la $\mathrm{VcM}$, se obtuvieron que todas son positivas y estadísticamente significativas $(p<.05)$, con una magnitud alta $\left(r_{s}>.50\right)$ para las dimensiones de violencia psicológica (entre .51 y .61), física leve (entre .54 y .59), sexual (entre .50 y .59) y daño (entre .50 y .55) (Vara-Horna \& López-Odar, 2016). Se observa que la dimensión instrumental del CUVINO presentó correlaciones significativas y moderadas con las dimensiones de VcM. Por otro lado, se aprecia que las dimensiones de violencia económica y física grave presentaron correlaciones significativas y moderadas con las dimensiones del CUVINO. Los resultados específicamente para estas correlaciones que resultaron significativas y moderadas, se debieron probablemente a que la VcM no evalúa el tipo de violencia instrumental. Asimismo, el CUVINO no evalúa el tipo de violencia económica ni agresión física grave de manera específica, es por ello que las correlaciones no arrojaron una magnitud alta, como en los demás casos. En los demás resultados, las correlaciones obtenidas fueron significativas $(\mathrm{p}<.05)$ y de magnitud alta $\left(r_{s}>.50\right)$. Cabe recalcar que el análisis de validez convergente permite corroborar las relaciones existentes entre los ocho factores del 
CUVINO y los seis factores de la escala de la VcM que también mide violencia en relaciones de pareja. Los resultados demuestran que las relaciones son altamente significativas en la muestra estudiada, y al ser altas indican que son asociaciones relevantes.

\section{Conclusiones}

Se concluye que el CUVINO cuenta con adecuadas propiedades psicométricas para su uso en la muestra de estudio al contar con evidencias de validez y confiabilidad. Se recomienda hacer un análisis de diferencias según el sexo de los participantes para poder determinar si el modelo del CUVINO es invariante entre los grupos. De esta manera, se podría concluir que ambos grupos funcionan de igual manera y evitar algún grupo de sesgo para un grupo específico.

Resulta conveniente indicar las limitaciones encontradas a partir de la realización de este estudio. Al ser un cuestionario de autoinforme, estos están sujetos a la deseabilidad social. Así mismo, el tipo de muestreo empleado para llevar a cabo el estudio fue no probabilístico (Hernández et al., 2014). Por consecuencia, se encuentra como limitación la generalización de los resultados, ya que se trata de una muestra con características específicas. Se sugiere que se realicen más validaciones en otros grupos muestrales para continuar con la acumulación de evidencias de validez del instrumento y la obtención de índices de ajuste absoluto dentro de los valores esperados.

\section{Referencias}

Abad, F., Olea, J., Ponsoda, V. \& García, C. (2011). Medición en ciencias sociales y de la salud. Madrid: Síntesis.

Ato, M., López, J. \& Benavente, A. (2013). Un sistema de clasificación de los diseños de investigación en psicología. Anales de Psicología, 29(3), 1038-1059. doi: 10.6018/analesps.29.3.178511

Beltrán, G., Albán, J., Zumba, I., Vera, J. \& Figueroa, M. (2018). Parejas violentas; análisis desde la perspectiva sistémica: una experiencia desde la práctica. Dilemas Contemporáneos: Educación, Política y Valores. Recuperado de http://www.dilemascontemporaneoseducacionpoliticayvalores.com/index.php/dilema s/article/view/330/815

Blum, D., Auné, S., Ahumada, C., Galibert, M. \& Attorresi, H. (2013). Criterios para la eliminación de ítems de un Test de Analogías Figurales. Summa Psicológica UST, 10(2), 49-56.

Byrne, B. M. (2006). Structural Equation Modeling with EQS: Basic Concepts, Applications, and Programming ( $2^{\mathrm{a}}$ ed.). New Jersey: Taylor Francis.

Carrascosa, L., Cava, M. J. \& Buelga, S. (2018). Perfil psicosocial de adolescentes españoles agresores y víctimas de violencia de pareja. Universitas Psychologica, 17(3), 1-10. doi: 10.11144/Javeriana.upsy17-3.ppae 
Cohen, J. (1988). Statistical power analysis for the behavioral sciences. Nueva York: Academic Press. Recuperado de https://books.google.es/books?hl=es\&lr=\&id=rEe0BQAAQBAJ\&oi=fnd\&pg=PP1\& $\mathrm{dq}=$ Statistical+Power+Analysis+for+the+Behavioral+Sciences+\&ots=swTQPsPOnb $\&$ sig=hMsK0jVMhr1JJJR-

RSMwA9YtlxY\#v=onepage\&q=Statistical\%20Power\%20Analysis\%20for\%20the\%2 0Behavioral\%20Sciences $\& \mathrm{f}=$ false

Cortés-Ayala, L., Flores Galaz, M., Bringas Molleda, C., Rodríguez-Franco, L., LópezCepero Borrego, J. \& Rodríguez Díaz, F. J. (2015). Relación de maltrato en el noviazgo de jóvenes mexicanos: análisis diferencial por sexo y nivel de estudios. Terapia Psicológica, 33(1), 5-12. doi: 10.4067/S0718-48082015000100001

Del Ángel, M. \& Barraza, A. (2017). Violencia mutua en el noviazgo: perfil psicosocial víctima-victimario en universitarios. Psicología y Salud, 27(2), 231-244.

Díaz, V., Feito, A., Díaz, F., González, M., Díaz, M. \& Pérez, A. (2013). Violencia de género en estudiantes de enfermería durante sus relaciones de noviazgo. Atención Primaria, 45(6), 290-296. doi: 10.1016/j.aprim.2012.11.013

Domínguez-Lara, S. (2019). Correlación entre residuales en análisis factorial confirmatorio: una breve guía para su uso e interpretación. Interacciones, 5(3), e207. doi: 10.24016/2019.v5n3.207

Fernández, A., Fuertes, A. \& Pulido, R. (2006). Evaluación de la violencia en las relaciones de pareja de los adolescentes. Validación del Conflict in Adolescent Dating Relationships Inventory (CADRI)-versión española. International Journal of Clinical and Health Psychology, 6(2), 339-358.

Gómez, M., Delgado, A. \& Gómez, Á. (2014). Violencia en relaciones de pareja de jóvenes y adolescentes. Revista Latinoamericana de Psicología, 46(3), 148-159. doi: 10.1016/S0120-0534(14)70018-4

Hernández, R., Fernández, C. \& Baptista, P. (2014). Metodología de la investigación. México: McGraw-Hill.

Hudson, W. \& McIntosh, S. (1981). The assessment of spouse abuse: Two quantifiable dimensions. Journal of Marriage and the Family, 43(4), 873-888. doi: 10.2307/351344

Instituto Nacional de Estadística e Informática [INEI]. (2019). Perú: indicadores de violencia familiar y sexual, 2012-2019. Recuperado de https://www.inei.gob.pe/media/MenuRecursivo/publicaciones_digitales/Est/Lib1686/ libro.pdf

Keith, T. Z. (2015). Multiple Regression and Beyond: An Introduction to Multiple Regression and Structural Equation Modeling ( $2^{\mathrm{a}}$ ed.). Nueva York: Routledge

Kline, P. (1986). A Handbook of Test Construction: Introduction to Psychometric Design. Nueva York: Methuen.

La República. (2019, noviembre 8). En Tacna el 86 \% de víctimas de violencia son mujeres y $14 \% \quad$ varones. La República. Recuperado de https://larepublica.pe/sociedad/2019/08/11/en-tacna-el-86-de-victimas-de-violenciason-mujeres-y-14-varones/ 
Li, C. H. (2016). Confirmatory factor analysis with ordinal data: Comparing robust maximum likelihood and diagonally weighted least squares. Behavior Research Methods, 48(3), 936-949. doi: 10.3758/s13428-015-0619-7

Lloret-Segura, S., Ferreres-Traver, A., Hernández-Baeza, A. \& Tomás-Marco, I. (2014). El análisis factorial exploratorio de los ítems: una guía práctica, revisada y actualizada. Anales de Psicología, 30(3), 1151-1169. doi: 10.6018/analesps.30.3.199361

Medrano, L. \& Muñoz-Navarro, R. (2017). Aproximación conceptual y práctica a los modelos de ecuaciones estructurales. Revista Digital de Investigación en Docencia Universitaria, 11(1), 216-236. doi: 10.19083/ridu.11.486.

Ministerio de la Mujer y Poblaciones Vulnerables [MIMP]. (2019). Informe Estadístico. Violencia en cifras. Recuperado de https://www.mimp.gob.pe/contigo/contenidos/publicararticulos/server/php/files/Informe-Estadistico-12-2019-Diciembre-2019.pdf

Muñiz, J. (2018). Introducción a la Psicometría. Madrid: Pirámide.

Observatorio Nacional de la Violencia Contra las Mujeres y los Integrantes del Grupo Familiar. (2018, diciembre 31). Tacna inaugura Observatorio de la violencia contra las mujeres y los integrantes del grupo familiar. Recuperado de https://observatorioviolencia.pe/tacna-inaugura-observatorio-de-la-violencia-contralas-mujeres-y-los-integrantes-del-grupo-familiar/

Ocampo, L. (2015). Autoestima y adaptación en víctimas de maltrato psicológico por parte de la pareja. Psicología desde el Caribe, 32(1), 145-168. doi: 10.14482/psdc.32.1.5204

Organización Mundial de la Salud [OMS]. (2002). Informe mundial sobre la violencia y la salud: resumen. Oficina Regional para las Américas de la Organización Mundial de la Salud. Washington: OPS. Recuperado de https://www.who.int/violence_injury_prevention/violence/world_report/es/summary_ es.pdf

Organización Mundial de la Salud [OMS]. (Ed.). (2013). Informe de la OMS destaca que la violencia contra la mujer es un problema de salud global de proporciones epidémicas. Recuperado de https://www.who.int/mediacentre/news/releases/2013/violence_against_women_2013 0620/es/

Rodríguez, L., Antuña, A., Rodríguez-Díaz, F. J. \& Herrero, F. J. (2007). Violencia de género en relaciones de pareja durante la adolescencia. Análisis diferencias del cuestionario de violencia entre novios (Cuvino). En R. Arce, F. Fariña, E. Alfaro, C. Civera \& F. Tortosa (Eds.), Psicología Jurídica. Violencia y Víctimas (pp. 137-146). Valencia: Diputación de Valencia.

Rodríguez, L., López, J., Rodríguez, F. J., Bringas, C., Antuña, M. \& Estrada, C. (2010). Validación del cuestionario de violencia entre novios (CUVINO) en jóvenes hispanohablantes: Análisis de resultados en España, México y Argentina. Anuario de Psicología Clínica y de la Salud, 6, 45-52.

Rodríguez, R., Riosvelasco, L. \& Castillo, N. (2018). Violencia en el noviazgo, género y apoyo social en jóvenes universitarios. Escritos de Psicología, 11(1), 1-9.

Rosseel, Y. (2012). Lavaan: An R package for structural equation modeling and more. Version 0.5-12 (BETA). Journal of Statistical Software, 48(2), 1-36. 
Rubio, F., López, M., Carrasco, M. \& Amor, P. (2017). Prevalencia de la violencia en el noviazgo: una revisión sistemática. Papeles del Psicólogo, 38(2), 135-147. doi: 10.23923/pap.psicol2017.2831

Ruiz, M. A., Pardo, A. \& San Martín, R. (2010). Modelo de ecuaciones estructurales. Papeles del Psicólogo, 31(1), 34-45.

Straus, M. (1979). Measuring intrafamily violence and conflict: the Conflict Tactics (CT) Scale. Journal of Marriage and Family, 41, 75-88. doi 10.2307/351733

Straus, M. (2008). Dominance and symmetry in partner violence by male and female university students in 32 nations. Children and Youth Services Review, 30(3), 252-275. doi: 10.1016/j.childyouth.2007.10.004

Tabachnick, B. \& Fidell, L. (2013). Using Multivariate Statistics (6 ${ }^{\circ}$ ed.). Northridge: California State University.

Vara-Horna, A. \& López-Odar, D. (2016). La violencia contra las mujeres en las universidades peruanas. Prevalencia e impacto en la productividad académica en las facultades deficiencias empresariales e ingenierías. Lima: GIZ \& USMP. Recuperado de

https://info.comvomujer.org.pe/catalogocomvo/productoscatalogos2016/21_ComVo Mujer_Estudio\%20de\%20Costos\%20en\%20Universidades\%20Peru_2016.pdf

Viladrich, C., Angulo-Brunet, A. \& Doval, E. (2017). A journey around alpha and omega to estimate internal consistency reliability. Anales de Psicología, 33(3), 755-782. doi: 10.6018/analesps.33.3.268401

Wu, A., Li, Z. \& Zumbo, B. (2007). Decoding the meaning of factorial invariance and updating the practice of multigroup confirmatory factor analysis: A demonstration with TIMSS data. Practical Assessment Research \& Evaluation, 12(3), 1-26. doi: $10.7275 / \mathrm{mhqa}-\mathrm{cd} 89$

Yanes, M., Hernández de la Cruz, A. \& Bautista, A. (2019). Violencia en el noviazgo en opinión de un grupo de adolescentes de la Sierra Norte de Puebla. Revista Chakiñan de Ciencias Sociales y Humanidades, 7, 25-39.

Contribución de los autores: a) Concepción y diseño del trabajo; b) Adquisición de datos; c) Análisis e interpretación de datos; d) Redacción del manuscrito; e) revisión crítica del manuscrito.

R. R. V. ha contribuido en a, b, d; E. M-M. en c, d, e.

Editora científica responsable: Dra. Cecilia Cracco. 